\title{
MIGRAÇÃO KIRCHHOFF EM PROFUNDIDADE DE SEÇÕES DE ONDAS PLANAS
}

\author{
Reynam C. Pestana ${ }^{1}$ e Celso A. Moreira Neto² \\ Recebido em 5 junho, 2006 / Aceito em 12 novembro, 2007 \\ Received on June 5, 2006 / Accepted on November 12, 2007
}

\begin{abstract}
We present a migration method for plane wave source data based on the Kirchhoff integral. Plane wave migration has been employed to increase the efficiency of the wavefield extrapolation. Point source records are synthesized into a plane wave and the resulting plane wave data can be migrated using the same scheme as the one for conventional shot-record migration. Plane wave migration reduces the migration computation time, because the extrapolation does not need to be done for all the individual shot records, but only for the constant plane wave sections. Here we have developed a version of the plane wave migration method based on the Kirchhoff integral. To calculate the traveltimes for the point source and also for plane wave we used a eikonal solver. Once all plane waves section are migrated independently of the others, the final image is obtained by stacking all common images gathers. Integral methods are easier than the wave equation methods to adapt to different recording geometries and also allow large and small models to be treated in the same way. Also, with the aid of the Asymptotic Ray Theory (ART), integral methods can be extended to handle arbitrary velocity structures without any dip limitation. In this paper the SEG-EAGE salt model example and a real dataset from Gulf of Mexico are used to test the theory and show that Kirchhoff plane wave depth migration can produce images comparables in quality to those obtained with Kirchhoff migration and also a reduced computation cost when comparable with the Kirchhoff migration of common shot gathers.
\end{abstract}

Keywords: pre-stack migration, Kirchhoff migration, plane wave, plane wave migration.

RESUMO. Recentemente novos métodos de migração utilizando ondas planas têm sido desenvolvidos com o intuito de aumentar a eficiência computacional da etapa de extrapolação dos campos de ondas durante o processo de migração. Nesses métodos os dados de fonte pontual são sintetizados em registros de fontes de ondas planas antes do processo de migração. Em seguida, os campos de ondas planas resultantes podem ser migrados usando qualquer um dos esquemas de extrapolação utilizados nas migrações convencionais de tiro comum. Assim, a migração de ondas planas consegue reduzir o número de extrapolações durante o processo de migração, uma vez que a extrapolação não é mais feita para todos os tiros individualmente, mas apenas para as seções de ondas planas sintetizadas. Na migração de ondas planas faz-se necessário também a continuação para baixo do campo de onda da fonte, que no caso é uma onda plana, bem como a depropagação dos campos de ondas planas sintetizados. Com este trabalho buscamos melhorar ainda mais a eficiência computacional do método de migração de ondas planas e, para tanto, estamos propondo a sua implementação através de operadores de propagação baseados na Teoria Assintótica do Raio. A nossa versão Kirchhoff de migração para dados sísmicos sintetizados em onda planas é implementada utilizando os tempos de trânsito tanto da onda plana como da fonte pontual, calculados através da solução da equação Iconal, usando-se a apropriada condição inicial. Também através do uso da Teoria Assintótica do Raio, a migração de ondas planas do tipo Kirchhoff pode ser estendida para tratar de meios com modelos de velocidades estruturalmente complexos, sem nenhuma limitação quanto à inclinação dos refletores. 0 novo método de migração Kirchhoff de dados de fonte de ondas planas é testado e sua aplicabilidade demonstrada usando-se os dados sintéticos 2D SEG-EAGE e também uma linha sísmica real do Golfo do México. Os resultados apresentados são bastante satisfatórios, comparáveis aos obtidos pela migração Kirchhoff, e conseguidos a um custo computacional menor do que 0 da migração Kirchhoff de tiro comum convencional.

Palavras-chave: Migração pré-empilhamento, migração Kirchhoff, ondas planas, migração de ondas planas.

\footnotetext{
${ }_{1}^{1}$ Departamento de Geofísica da Terra e do Meio Ambiente, Instituto de Física/CPGG, Universidade Federal da Bahia, Campus Universitário da Federação, 40170-290 Salvador, BA, Brasil. Tel.: (71) 3283-8521; Fax: (71) 3283-8551 - E-mail: reynam@cpgg.ufba.br

2 Petróleo Brasileiro S.A. Unidade de Negócios Sergipe/Alagoas (UN-SEAL), Rua Acre, 2504, Siqueira Campos, 49080-010 Aracaju, SE, Brasil. Tel.: (79) $3212-3292$ -E-mail: celsoamn@petrobras.com.br
} 


\section{INTRODUÇÃo}

A migração é uma das principais etapas do processamento de dados sísmicos que, através da extrapolação do campo de ondas, corrige os efeitos ocorridos durante a propagação do pulso sísmico, coloca as reflexões em suas verdadeiras posições espaciais, colapsa as difrações possibilitando uma melhor interpretação dos dados sísmicos.

Este procedimento pode ser formulado em termos de dados de pontos de tiro comum, onde cada tiro registrado é migrado separadamente e a seção da imagem final é obtida somando-se os tiros migrados individualmente. Considera-se, neste caso, que a fonte sísmica é uma fonte pontual.

A migração sísmica de ponto de tiro comum pré-empilhamento, baseada na equação da onda normalmente produz imagens de melhor qualidade do que as migrações do tipo Kirchhoff (MK). Entretanto, esse processo requer uma alta demanda computacional e tem o seu uso limitado no processamento de dados sísmicos 3D.

Mais recentemente, os métodos de migração utilizando ondas planas têm sido bastante empregados visando aumentar a eficiência computacional da extrapolação dos campos de ondas (Rietveld et al., 1994; Duquet et al., 2001; Pestana et al., 2001; Liu et al., 2002; Zhang et al., 2003; Zhang et al., 2005; Moreira Neto et al., 2005). Nesses métodos os registros de fonte pontual são sintetizados em dados sísmicos de fonte de onda plana, antes do processo de migração. Essas técnicas de migração também são conhecidas como migrações do tipo "Phase-encoding" (Morton \& Ober, 1998), cuja função peso é uma onda plana, que é usada para comprimir os dados sísmicos a serem migrados. Desta forma, o campo de fonte de onda plana resultante pode ser migrado usando qualquer um dos esquemas utilizados nas migrações convencionais de tiro comum. Portanto, a migração de ondas planas assim implementada consegue reduzir o número de extrapolações, durante o processo de migração, uma vez que a extrapolação não é aplicada a todos os tiros individualmente, mas apenas às respectivas seções de onda plana sintetizadas. Além disso, a eficiência computacional do método de migração de ondas planas pode ser melhorada através do uso de operadores de propagação baseados na Teoria Assintótica do Raio, como nas migrações convencionais do tipo Kirchhoff.

Atualmente os métodos de migração Kirchhoff são os métodos mais usados no processamento de dados sísmicos, principalmente na migração de dados 3D, em razão da sua flexibilidade de aplicação a diferentes geometrias de aquisição e também a pequenos ou grandes volumes de dados indiferente- mente. Além disso, eles podem fornecer imagens sísmicas de subsuperfície de boa qualidade. Entretanto, a sua aplicação em meios geologicamente complexos requer o cálculo dos tempos de várias chegadas ("multi-path arrivals") de forma a permitir que as MK possam imagear mais adequadamente os dados sísmicos provenientes de áreas estruturalmente complexas.

Na realização da migração de ondas planas faz-se necessário a continuação para baixo do campo de onda da fonte, que neste caso é uma fonte de onda plana, bem como a depropagação dos campos de ondas planas sintetizados. E para melhorar ainda a eficiência computacional do método de migração de ondas planas, estamos propondo neste trabalho a implementação da etapa de extrapolação através de operadores de propagação construídos com base na teoria do raio.

Portanto, este trabalho tem como objetivo apresentar uma versão 2D do tipo Kirchhoff para a migração de dados sísmicos sintetizados em onda planas. E para tanto, os tempos de trânsito, tanto das ondas planas como das fontes pontuais, serão calculados através da solução da equação Iconal (Faria \& Stoffa, 1994), tornando este método computacionalmente eficiente e abrindo a possibilidade de futuras aplicações deste tipo de migração em dados sísmicos 3D.

0 método aqui proposto foi testado nos dados sintéticos SEG-EAGE e também em uma linha sísmica do Golfo do México. As migrações apresentaram resultados bastante satisfatórios e foram conseguidos a um menor custo computacional, em relação às migrações convencionais de tiro comum.

\section{MIGRAÇÃO DE ONDAS PLANAS}

A migração de seções de tiro comum é implementada através da continuação independente de tiros e receptores e subseqüente aplicação da condição de imagem (Aldunate, 2002).

Como os operadores de extrapolação são operadores lineares que atuam sobre os campos de ondas e realizam a continuação desses campos, a combinação linear de vários tiros resulta em um campo de onda que pode ser continuado para baixo pelos mesmos operadores de extrapolação (Moreira Neto, 2004).

A imagem sísmica fornecida através dessa técnica de migração é obtida tomando-se o atraso ("lag") zero da correlação cruzada desses campos, dada pela seguinte relação:

$$
I(x, z)=\sum_{\omega} \tilde{S}^{*}(\omega, x, z) \tilde{R}(\omega, x, z)
$$

onde $\tilde{S}^{*}(\omega, x, z)$ representa 0 complexo conjugado da combinação linear de vários tiros e $\tilde{R}(\omega, x, z)$ a seção de onda plana 
extraída dos receptores e dados por:

$$
\begin{aligned}
& \tilde{S}(\omega, x, z)=\sum_{j=1}^{N} a_{j}(\omega) S_{j}(\omega, x, z) \\
& \tilde{R}(\omega, x, z)=\sum_{j=1}^{N} a_{j}(\omega) R_{j}(\omega, x, z)
\end{aligned}
$$

onde, agora, $S_{j}(\omega, x, z)$ e $R_{j}(\omega, x, z)$ são, respectivamente, os campos de ondas da fonte e dos receptores do j-ésimo tiro e $a_{j}(\omega)$, com $j=1,2, \ldots, N$, é uma função peso. Substituindose as equações (2) e (3) em (1), tem-se:

$$
\begin{gathered}
I(x, z)= \\
\sum_{j=1}^{N} \sum_{\omega}\left|a_{j}(\omega)\right|^{2} S_{j}^{*}(\omega, x, z) R_{j}(\omega, x, z)+ \\
\sum_{j \neq k}^{N} \sum_{\omega} a_{j}^{*}(\omega) a_{k}(\omega) S_{j}^{*}(\omega, x, z) R_{k}(\omega, x, z)
\end{gathered}
$$

Escolhendo-se $\left|a_{j}(\omega)\right|^{2}=1$, o primeiro termo da equação (4) torna-se exatamente a soma das migrações dos diversos tiros. 0 segundo termo, representa a correlação cruzada dos campos de ondas de diferentes tiros e receptores, o qual é considerado ruído (artefato), pois não existe na migração convencional de tiro comum. Desta forma, os termos $a_{j}(\omega)$ devem ser escoIhidos com o objetivo de minimizar a contribuiçãa desses termos cruzados no resultado do processo de imageamento.

\section{Composição de ondas planas}

Para compor a fonte de onda plana, com parâmetro de raio $p$, a função peso $a_{j}(\omega), \operatorname{com} j=1,2, \ldots, N$, é definida como uma onda plana, que é dada por

$$
a_{j}(\omega)=e^{i \omega p\left(x_{j}-x_{o}\right)},
$$

onde $p$ define a direção de propagação da onda plana e $x_{0}$ a posição do primeiro tiro. Substituindo-se (5) em (4) obtém-se a condição de imagem para a migração de seções de ondas planas constantes. Desta forma, a imagem final é construída após 0 empilhamento de todas as seções de ondas planas migradas. Segundo Liu et al. (2002), desprezando-se os termos cruzados, a seção final migrada de cada onda plana, passa a ter a seguinte forma:

$$
I(x, z) \cong \sum_{j=1}^{N} \sum_{\omega} S_{j}^{*}(\omega, x, z) R_{j}(\omega, x, z)
$$

A equação (6) é exatamente a condição de imagem usada na migração de tiro comum convencional. Portanto, a migração de seções de ondas planas pode ser considerada um procedimento equivalente à migração de seção de tiro comum.

Além disso, essa técnica de migração possibilita 0 aumento da eficiência computacional da migração de tiro comum, pois vários tiros são empilhados com diferentes pesos (ondas planas) e podem ser migrados de uma única vez. Entretanto, um número suficiente de seções de parâmetros de raio devem ser usados para suprimir ou diminuir os artefatos, devido aos termos cruzados que aparecem na equação (4).

\section{Migração de Ondas Planas - Método Kirchhoff}

A integral de Kirchhoff (Schneider, 1978) para a continuação dos campos de ondas das fontes e dos receptores em profundidade é dado por:

$$
\begin{gathered}
P(\mathrm{x}, \omega)= \\
\int \partial_{n} G(\mathrm{x}, \mathrm{s}) d \mathrm{~s} \int \partial_{n} G(\mathrm{x}, \mathrm{g}) P(\mathrm{~s}, \mathrm{~g}, \omega) d \mathrm{~g}
\end{gathered}
$$

onde $P(\mathrm{~s}, \mathrm{~g}, \omega)$ é 0 campo de ondas registrado na superfície, s é vetor posição da fonte, $g$ é o vetor posição do receptor, $G$ é função de Green, $\partial_{n} G$ é a derivada normal à superfície da função de Green, $x$ é 0 vetor posição do espalhador em subsuperfície, $\omega$ é a freqüência angular e $P(\mathrm{x}, \omega)$ é 0 campo de ondas extrapolado em profundidade.

Para extrapolar o campo de ondas registrado $P(\mathrm{~s}, \mathrm{~g}, \omega)$ precisamos construir a função de Green. Para meios heterogêneos a função de Green é, normalmente, construída usando-se a Teoria Assintótica do Raio (ART). 0 operador de continuação para baixo, representado pela função de Green com aproximação ART é dado por:

$$
P(\mathrm{x}, \mathrm{s})=A(\mathrm{x}, \mathrm{s}) e^{i \omega t(\mathrm{x}, \mathrm{s})}
$$

onde $A(\mathrm{x}, \mathrm{s})$ é 0 termo de amplitude e $t(\mathrm{x}, \mathrm{s})$ é 0 tempo de trânsito, dado pela equação Iconal.

Usando a função de Green com aproximação de alta freqüência ART e supondo que a amplitude não varia muito com a distância (Hildebrand \& Carroll, 1993), então a equação (7) pode ser reescrita como

$$
\begin{gathered}
P(\mathrm{x}, \omega)=-\omega^{2} \int d \mathrm{~s} \\
\times \int W(\mathrm{x}, \mathrm{s}, \mathrm{g}) e^{i \omega[t(\mathrm{x}, \mathrm{s})+t(\mathrm{x}, \mathrm{g})]} P(\mathrm{~s}, \mathrm{~g}, \omega) d \mathrm{~g}
\end{gathered}
$$

onde $t(\mathrm{x}, \mathrm{S})$ and $t(\mathrm{x}, \mathrm{g})$ são os tempos de trânsito a partir da fonte e do receptor ao ponto espalhador $x$, respectivamente, e 0 
termo $e^{i \omega[t(\mathrm{x}, \mathrm{s})+t(\mathrm{x}, \mathrm{g})]}$ é definido como 0 operador de atraso e a função $W(x, s, g)$, definida por:

$$
W(\mathrm{x}, \mathrm{s}, \mathrm{g})=\partial_{n} t(\mathrm{x}, \mathrm{s}) A(\mathrm{x}, \mathrm{s}) \partial_{n} t(\mathrm{x}, \mathrm{g}) A(\mathrm{x}, \mathrm{g}) \text {. }
$$

Para obter o operador de continuação do campo de ondas planas, realizamos a decomposição dos dados registrados $P(\mathrm{~s}, \mathrm{~g}, \omega)$, em seus componentes de ondas planas através da transformada "slant-stack". Isto é obtido aplicando-se um atraso no tempo a cada tiro (Schultz \& Claerbout, 1978). Em outras palavras, cada família de receptor comum é transformada em ondas planas usando-se:

$$
P\left(\mathrm{p}_{\mathrm{s}}, \mathrm{g}, \omega\right)=\int d \mathrm{~s} P(\mathrm{~s}, \mathrm{~g}, \omega) \exp \left[-i \omega\left(\mathrm{p}_{\mathrm{s}} \cdot \mathrm{s}\right)\right],
$$

onde $P\left(p_{s}, g, \omega\right)$ representa 0 campo de onda plana referente ao vetor parâmetro de raio $p_{s}$.

No domínio da freqüência a transformada "slant-stack" pode ser vista como uma transformada de Fourier, fazendo-se $k=\omega p$. Então, a transformada "slant-stack" inversa é dada por:

$$
\begin{gathered}
P(\mathrm{~s}, \mathrm{~g}, \omega)= \\
\omega^{2} \int d \mathrm{p}_{\mathrm{s}} P\left(\mathrm{p}_{\mathrm{s}}, \mathrm{g}, \omega\right) \exp \left[i \omega\left(\mathrm{p}_{\mathrm{s}} \cdot \mathrm{s}\right)\right] .
\end{gathered}
$$

Expandindo o tempo de trânsito da fonte $t(\mathrm{x}, \mathrm{s})$ em termos do vetor vagarosidade e do vetor posição do ponto de espalhamento (Akbar et al., 1996), como segue:

$$
t(x, s)=p_{S}^{\prime} \cdot(x-s),
$$

onde $p_{S}^{\prime}$ é 0 vetor vagarosidade que fornece a direção do raio a partir da fonte até o ponto espalhador.

Em seguida, substituindo-se as equações (12) e (13) na equação (9), assumindo que a amplitude e a derivada normal não variam com a distância, ou seja, que podemos escrever que $W(\mathrm{x}, \mathrm{s}, \mathrm{g})=W(\mathrm{x})$, e resolvendo-se as integrais com relação a s e $p_{s}$, obtemos:

$$
\begin{gathered}
P\left(\mathrm{x}, \mathrm{p}_{\mathrm{s}}^{\prime}, \omega\right)= \\
-\omega^{2} W(\mathrm{x}) \int e^{i \omega\left[t(\mathrm{x}, \mathrm{g})+\mathrm{p}_{\mathrm{s}}^{\prime} \cdot \mathrm{x}\right]} P\left(\mathrm{p}_{\mathrm{s}}^{\prime}, \mathrm{g}, \omega\right) d \mathrm{~g}
\end{gathered}
$$

Para aplicar a condição de imagem calculamos a transformada inversa de Fourier da equação anterior na freqüência. No domínio do tempo a equação (14) é dada por:

$$
\begin{gathered}
P\left(\mathrm{x}, \mathrm{p}_{\mathrm{S}}^{\prime}, \tau\right)= \\
W(\mathrm{x}) \int \frac{\partial^{2}}{\partial \tau^{2}} P\left(\mathrm{p}_{\mathrm{S}}^{\prime}, \mathrm{g},\left[\tau+t(\mathrm{x}, \mathrm{g})+\mathrm{p}_{\mathrm{S}}^{\prime} \cdot \mathrm{x}\right]\right) d \mathrm{~g}
\end{gathered}
$$

onde $\tau$ é tempo de interseção (Stoffa et al., 1981).

Após a aplicação da condição de imagem, $\tau=0$, a equação (15) se reduz a

$$
P\left(\mathrm{x}, \mathrm{p}_{\mathrm{s}}^{\prime}\right)=W(\mathrm{x}) \int U\left(\mathrm{p}_{\mathrm{s}}^{\prime}, \mathrm{g},\left[t(\mathrm{x}, \mathrm{g})+\mathrm{p}_{\mathrm{s}}^{\prime} \cdot \mathrm{x}\right]\right) d \mathrm{~g},
$$

onde

$$
U\left(p_{s}^{\prime}, g, \tau\right)=\frac{\partial^{2}}{\partial \tau^{2}} P\left(p_{s}^{\prime}, g, \tau\right) .
$$

Note que a quantidade $t(\mathrm{x}, \mathrm{g})$, que é 0 tempo de trânsito do ponto espalhador ao receptor, pode ser calculada simulandose uma fonte pontual a partir da posição do receptor. A quantidade $p_{S}^{\prime} \cdot x$ é obtida através do cálculo do tempo de chegada da fonte plana. Logo, precisamos conhecer apenas a posição do receptor e do espalhador para calcular $t(x, g)$ e $p_{s}^{\prime} \cdot x$. 0 termo $\left[t(x, g)+p_{S}^{\prime} \cdot x\right]$ é 0 tempo de atraso que será aplicado a cada onda plana, durante o processo de migração.

Portanto, a equação (16) representa a integral de Kirchhoff para a continuação de campos de ondas planas. E neste método integral um atraso no tempo, baseado na distância da fonte a um ponto de referência, é usado para combinar, no domínio da freqüência, diferentes registros de tiro comum. Então, cada campo de onda plana sintetizado (seção $p_{S}^{\prime}$ ) é o resultado da superposição de todos os tiros do experimento sísmico. Como no método "Areal Shot" (Romero et al., 2000) ou "Delayed-shot Migration Method" (Liu et al., 2002; Zang et al., 2003), que são também métodos de migração de ondas planas baseados em soluções da equação da onda, precisamos somar um número razoável de seções de ondas planas migradas, para reduzir a influência dos termos-cruzados e conseguir uma imagem de boa qualidade de todo o modelo de subsuperfície.

\section{Meios Planos Estratificados}

Como a transformada "slant-stack", também chamada de transformada tau-p, é independente da velocidade do modelo, o tempo de atraso $\left(p_{s}^{\prime} \cdot x\right)$ é o único termo na equação (16) a ser modificado. Em particular, para um modelo 2D, com apenas variação vertical de velocidade, $v=v(z)$, 0 tempo de trânsito da onda plana é obtido somando-se os tempos de percurso ao longo do caminho do raio, dentro de cada camada, e é dado pela seguinte expressão:

$$
t\left(p^{\prime}, x_{g}\right)=p^{\prime}\left(x-x_{g}\right)+\int_{0}^{z} \sqrt{1 / v(z)^{2}-p^{\prime 2}} d z
$$

Assim, no caso 2D, a equação (16) é reescrita como:

$$
\begin{gathered}
P\left(x, z, p^{\prime}\right)= \\
W(x) \int U\left[p^{\prime}, x_{g}, T\left(p^{\prime}, x, z, x_{g}\right)\right] d x_{g}
\end{gathered}
$$


onde,

$$
\begin{gathered}
T\left(p^{\prime}, x, z, x_{g}\right)=p^{\prime}\left(x-x_{g}\right)+ \\
+\int_{0}^{z^{\prime}} \sqrt{1 / v\left(z^{\prime}\right)^{2}-p^{\prime 2}} d z^{\prime}+t\left(x, x_{g}\right)
\end{gathered}
$$

A imagem final migrada é obtida após a soma de todas as seções de ondas planas constantes migradas. Assim, temos que:

$$
P(x, z)=\int P\left(x, z, p^{\prime}\right) d p^{\prime}
$$

\section{EFICIÊNCIA DO MÉTODO KIRCHHOFF DE ONDAS PLANAS}

A nossa motivação em apresentar um método Kirchhoff de ondas planas é melhorar ainda mais a eficiência computacional dos métodos de migração de ondas planas. Então, iremos comparar 0 custo da migração de ondas planas Kirchhoff 2D com 0 custo da migração Kirchhoff de famílias de tiro comum. Considerandose $N_{x_{s}}$ tiros em uma linha sísmica com espaçamento entre tiros de $\Delta x_{s}$, espaçamento $\Delta x_{r}$ entre receptores e $\mathrm{L}$ a abertura máxima do operador de migração, então, o custo computacional da migração Kirchhoff de tiro comum $\left(C_{T C}\right)$ é proporcional ao número de posiç̧̃̃es de receptores dentro da abertura multiplicado pelo número de tiros $N_{x_{s}}$, isto é,

$$
C_{T C}=\frac{K N_{x_{s}} L}{\Delta x_{r}},
$$

onde $K$ é uma constante que representa o valor médio referente ao número de operações aritméticas efetuadas em cada ponto da malha do modelo vezes o número de profundidades migradas.

No caso da migração Kirchhoff de ondas planas, temos que $N_{p}$ seções de ondas planas serão migradas tomando toda a extensão da linha sísmica, com o número de posições dos receptores determinado por $N_{x_{s}} \Delta x_{s} / \Delta x_{r}$. Desde que o número de operações por ponto da malha é a mesma da migração Kirchhoff de tiro comum, ou seja, o número $\mathrm{K}$ tem 0 mesmo valor da migração de tiro comum, logo o custo computacional da migração de ondas planas tipo Kirchhoff proposto é

$$
C_{O P}=\frac{K N_{p} N_{x_{s}} \Delta x_{s}}{\Delta x_{r}}
$$

A razão entre as relações (21) e (22) fornece a rapidez da migração de ondas planas, para 0 caso $2 \mathrm{D}$, com relação à migração de tiro comum Kirchhoff convencional:

$$
R_{2 D}=\frac{C_{T C}}{C_{O P}}=\frac{L}{N_{p} \Delta x_{s}}
$$

Para 0 dado SEG-EAGE, que possui uma geometria típica 2D, e se considerarmos a abertura $L=6750 m, N_{p}=60 \mathrm{e}$ $\Delta x_{s}=20 \mathrm{~m}$, a relação (23) fornece uma razão aproximadamente igual a 5,6, indicando que o método de migração de ondas planas Kirchhoff é cerca de 5,6 vezes mais rápido do que a migração Kirchhoff de tiro comum.

\section{AMOSTRAGEM DOS PARÂMETROS DE RAIO}

Mostramos inicialmente que a transformada "slant-stack" pode ser vista com uma transformada de Fourier, apenas substituindose $k_{s}$ por $\omega p$. Desta forma, a transformada "slant-stack" obedece as mesmas regras de amostragem aplicadas a transformada discreta de Fourier.

0 significado físico do parâmetro $p$ usado na transformada "slant-stack" vem da relação $\Delta t=p \Delta x$; isto é, 0 atraso no tempo, $\Delta t$, entre dois tiros adjacentes separados por $\Delta x$. Assim,

$$
p=\frac{\Delta t}{\Delta x_{s}}=\frac{\sin \alpha_{s}}{v_{s}}
$$

onde $\alpha_{s}$ é 0 ângulo de saída do raio, relativo à vertical e $v_{s}$ a velocidade na posição do tiro.

Para garantir que o resultado da transformada "slant-stack" represente suficientemente 0 dado original, temos que:

$$
\omega \Delta p \leq \Delta k_{s}=\frac{2 \pi}{N_{S} \Delta x_{S}}
$$

onde $N_{s}$ é o número de tiros dentro de uma seção de receptor comum, $\Delta x_{s}$ é 0 espaçamento entre tiros, tal que $N_{s} \Delta x_{s}$ é 0 comprimento total de uma seção de receptor comum. Para uma faixa de ângulos de saída, $\alpha_{1} \leq \alpha_{s} \leq \alpha_{2}$ e $N_{p}$ seções de parâmetro de raio, temos que

$$
\Delta p=\frac{\sin \alpha_{2}-\sin \alpha_{1}}{v_{s} N_{p}}
$$

Substituindo a equação (26) em (25), podemos estimar 0 número de seções de $p$, como:

$$
N_{p} \geq \frac{N_{s} \Delta x_{s} f\left(\sin \alpha_{2}-\sin \alpha_{1}\right)}{v_{s}}
$$

onde $f=\omega / 2 \pi$. Dessa desigualdade verificamos que 0 número de seções de parâmetro de raio depende da freqüência. Por exemplo, para uma seção de receptores comuns de comprimento $N_{s} \Delta x_{s}=6000 \mathrm{~m}$, ângulo de saída menor ou igual a $30^{\circ}$ e $v_{s}=1500 \mathrm{~m} / \mathrm{s}$, a partir da desigualdade (27) obtemos aproximadamente 60 seções de $p$, para uma freqüência dominante de $15 \mathrm{~Hz}$. 
Assim, aplicando a teoria da amostragem para a transformada "slant-stack", mostramos que a desigualdade (27) fornece o número mínimo de seções de parâmetros de raio que devemos usar para preservar toda a informação de cada seção de receptor comum.

\section{RESULTADOS NUMÉRICOS Aplicação em Dados Sintéticos}

0 modelo em profundidade mostrado na Figura 1(a) representa uma estrutura simples, com camadas plano-horizontais, parcialmente seccionadas por uma falha. Na zona da falha diferentes mergulhos são observados.

Para testar o método de migração Kirchhoff de ondas planas dados sintéticos foram gerados a partir desde modelo através de modelagem no domínio $x$-t, usando-se o programa "Susynlv" do pacote "Seismic Unix" (SU) do "Center Wave Phenonema - Colorado School of Mines". 0 campo de velocidades em profundidade, Figura 1(b), empregado na geração dos dados foi criado com o programa "Makevel", também do pacote SU, utilizando-se um gradiente de velocidade tanto vertical como horizontal.

(a)
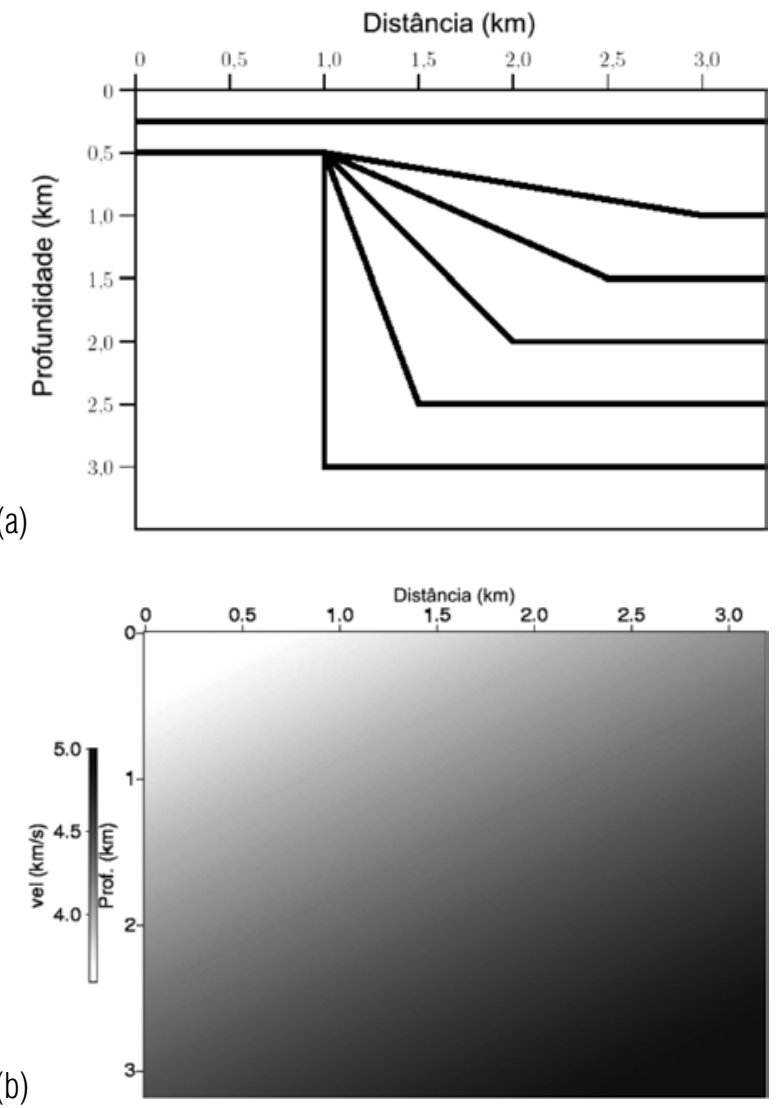

Figura 1 - (a) Modelo em profundidade; (b) Campo de velocidade. Este campo apresenta gradiente de velocidade vertical e horizontal.
Geramos 200 tiros que foram ordenados em famílias de receptores comuns e decompostos em seções de ondas planas (eq. 11). Selecionamos 61 seções de parâmetros de raio, variando de $-0,3 \mathrm{~s} / \mathrm{km}$ a $0,3 \mathrm{~s} / \mathrm{km}$, para testar 0 algoritmo de migração de ondas planas implementado.

0 resultado da migração Kirchhoff de fonte de ondas planas é mostrado na Figura 2(a) e o resultado com a migração Kirchhoff convencional de tiro comum, programa Sukdmig2d do pacote SU, é apresentado na Figura 2(b).

0 resultado da migração Kirchhoff de tiro comum apresenta um resultado mais limpo, enquanto o resultado obtido com método de ondas planas, usando apenas 61 seções, ainda conseguiu uma seção migrada final de boa qualidade. Desses resultados observa-se que as imagens produzidas pelos dois métodos apresentam os refletores corretamente posicionados, em conformidade com o modelo em profundidade, Figura 1(a).

(a)
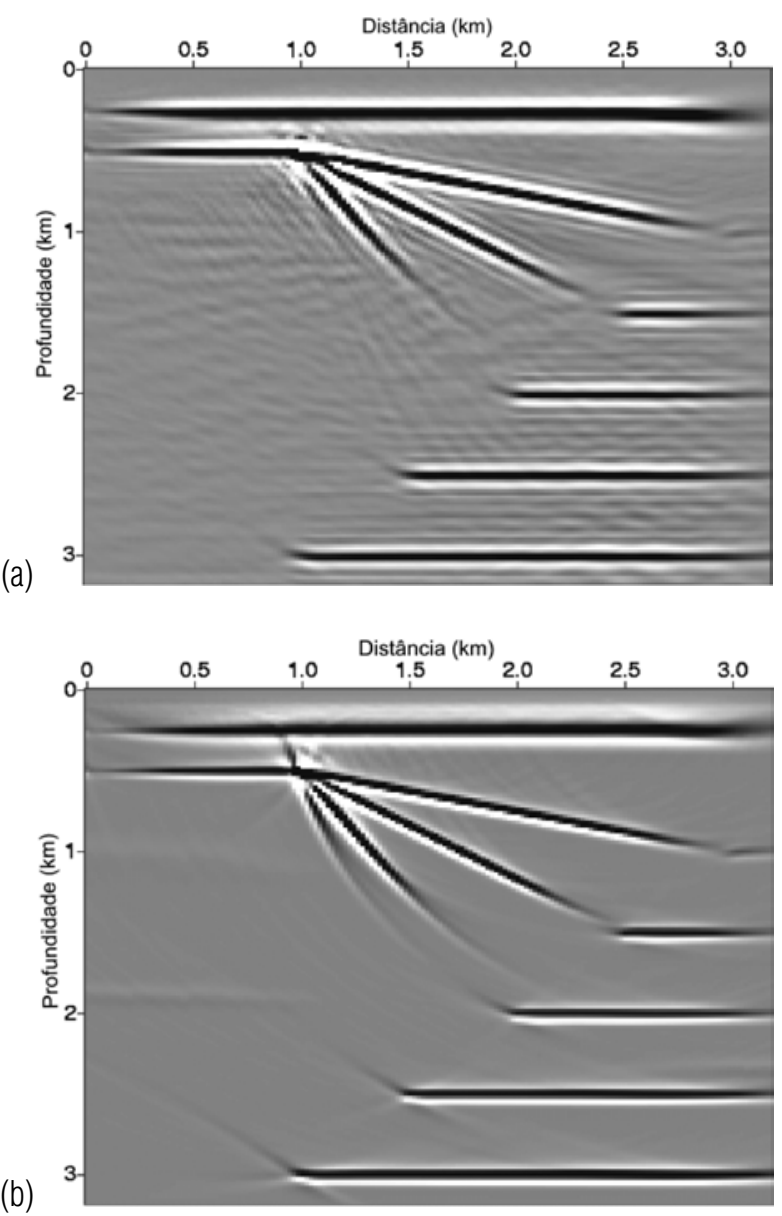

Figura 2 - (a) Resultado da migração de fonte de onda plana. Este resultado foi conseguido somando-se 61 seções de parâmetros de raio; (b) Resultado da migração Kirchhoff de 200 tiros com o pacote "Seismic Unix". 


\section{Dados SEG-EAGE}

0 modelo SEG-EAGE 2D representa uma almofada de sal associada a uma configuração espacial de refletores e falhamentos com razoável complexidade estrutural. Este dado sintético foi modelado através de diferenças finitas, a partir do campo de velocidades mostrado na Figura 3. Ele possui 625 tiros, 240 receptores espaçados de $20 \mathrm{~m}$ e com espaçamento entre tiros também de $20 \mathrm{~m}$. 0 campo de velocidades intervalares possui uma malha de 675 pontos na horizontal por 210 pontos na direção vertical, com espaçamento de $20 \mathrm{~m}$ em ambas as direções.

Este modelo é um excelente teste para os métodos de migração, pois a forma irregular da almofada de sal, combinada com 0 forte contraste de velocidade entre 0 sal e o meio circundante, causa sérios problemas na construção da imagem em profundidade. As porções do modelo que merecem especial atenção na avaliação dos métodos de migração são: os contornos do corpo de sal, as falhas subverticais abaixo da almofada de sal, a estrutura anticlinal com ápice nas coordenadas $x=4 \mathrm{~km} \mathrm{e} z=$ $3,6 \mathrm{~km}$ e 0 refletor horizontal localizado a 3,8 km de profundidade. Todas essas feições são de difícil imageamento, por estarem situadas abaixo do corpo de sal.

Para a aplicação do método Kirchhoff de ondas planas, os tiros originais foram ordenados em seções de receptor comum e em seguida transformados em seções de fontes de ondas planas, usando-se a transformada "slant-stack". Para esses dados foram geradas 81 seções de ondas planas de $-0,4 \mathrm{~s} / \mathrm{km}$ a 0,4 s/km, com intervalo de $0,01 \mathrm{~s} / \mathrm{km}$. Os tempos de trânsito, tanto de onda plana como de fonte pontual, foram calculados a partir do método de solução da equaç̃ão Iconal por diferenças finitas (Schneider et al., 1992). Todas as seções de ondas planas foram migradas separadamente e a seção final é obtida somando-se todas as seções migradas. A Figura 4(a) mostra 0 resultado da nossa migração Kirchhoff de fontes de ondas planas, e, para efeito de comparação, também o resultado obtido com o método Kirchhoff convencional de tiro comum, usando-se todos os 625 tiros, Figura 4 (b).

Também mostramos na Figura 5(a) as famílias de imagem comum ("Common image gathers" - CIG) desses dados, geradas a partir do nosso método Kirchhoff de migração de ondas planas e na Figura 5(b) destacamos os CIG nas posições 2,3,6,7,11 e 12 km, respectivamente. Nessas seções observamos que as reflexões se apresentam horizontalizadas, independente do valor do parâmetro do raio, já que a velocidade usada na migração foi idêntica à da modelagem dos dados. Já nas posições 3,6 e $7 \mathrm{~km}$ os refletores não ficaram completamente horizontalizados. Nesta parte do modelo de velocidades, almofada de sal, os tempos calculados, usando apenas as primeiras chegadas, não fornecem uma boa imagem do topo do sal.

As imagens apresentadas pelas migrações Kirchhoff convencional e de ondas planas produziram imagens muito parecidas, conseguindo imagear os flancos, topo e base da almofada de sal e parte dos refletores abaixo da profundidade de $2,5 \mathrm{~km}$. Porém, as regiões indicadas por A, B e C, na Figura 4(a), resultado da migração de ondas planas, ficaram com uma qualidade inferior à migração Kirchhoff de tiro comum (Figura 4b). E quanto ao custo computacional, a migração Kirchhoff de ondas planas foi cerca de 6 vezes mais rápida do que a migração Kirchhoff convencional.

Podemos ainda notar partir dos resultados que as migrações não conseguem recuperar completamente as estruturas abaixo da almofada de sal, o que é extremamente difícil e ainda se constitui em um dos principais desafios para as técnicas de migração em profundidade.

Podemos acrescentar que o método de migração de onda planas produz naturalmente seções de imagem comum em função do parâmetro do raio, que podem ser utilizadas para a análise do campo de velocidades ou também como seções de entrada para um procedimento de análise de AVO/AVA, mas para isso a migração precisaria ser de amplitude verdadeira. Essas seções de família de imagem comum são indexadas pelo ângulo da onda plana na superfície e são computadas mais eficientemente dos que as famílias de imagem comum fornecidas pelos métodos de migração de tiro comum. A geração das famílias de imagem comum, indexadas pelo ângulo de saída da onda plana na superfície, sem nenhum custo computacional adicional, é uma das grandes vantagens, do ponto de vista prático, desse novo método de migração Kirchhoff de ondas planas.

\section{Dados do Golfo do México}

Também aplicamos nosso método de migração de seções de ondas planas a uma linha sísmica real do Golfo do México. Esses dados marítimos são do campo de petróleo "Mississippi Canyon", Iocalizado no Golfo do México, e foram adquiridos em uma região que se caracteriza pela presença de um corpo de sal achatado e também por estratificações horizontais ao seu redor e abaixo do corpo de sal. A Figura 6(a) ilustra o modelo de velocidades que usamos na migração de ondas planas, que foi gentilmente cedido pelo Projeto SEP da Universidade de Stanford.

A linha sísmica marítima 2D que migramos contém 1001 tiros, espaçados de 26,67 m, onde cada família de tiro é formada por 180 receptores, também espaçados de 26,67 m. Cada traço tem 6,0 $\mathrm{s}$ de registro, amostrados a cada $4 \mathrm{~ms}$, ou seja, um total de 


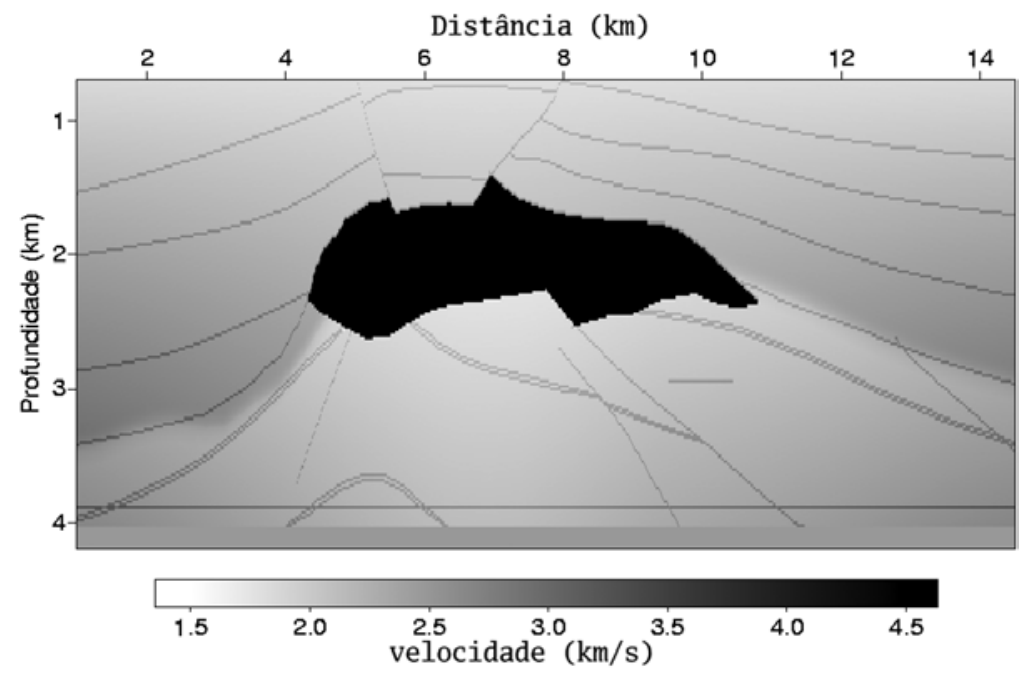

Figura 3 - Modelo de velocidade dos dados SEG-EAGE.

(a)
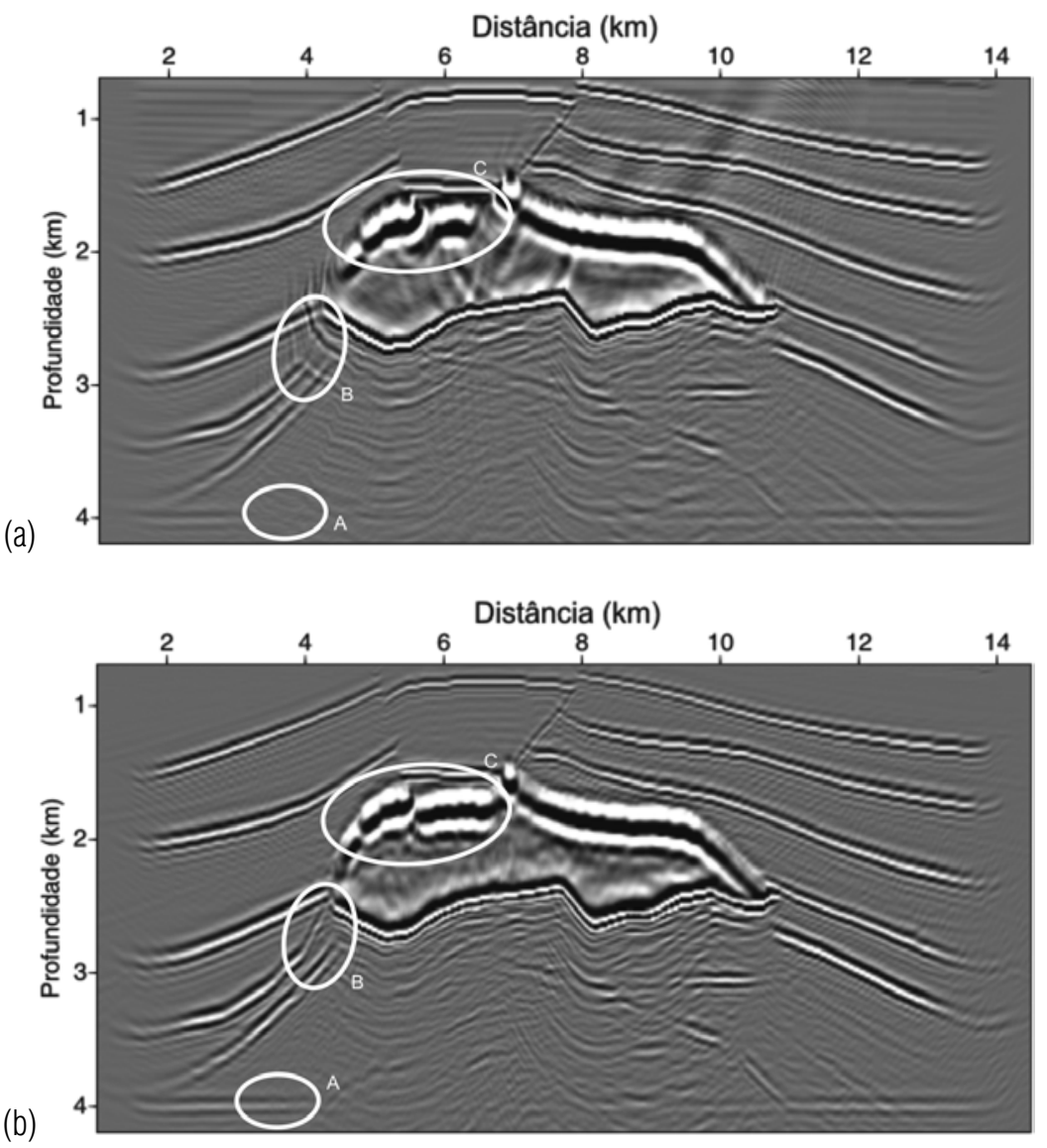

Figura 4 - (a) Resultado da migração Kirchhoff de ondas planas com 81 seções de parâmetro de raio (-0,4 a $0,4 \mathrm{~s} / \mathrm{km}$, com incremento de 0,01 s/km). (b) Resultado da migração Kirchhoff de tiro comum, de todos os 625 tiros, usando-se o pacote "Seismic Unix" (SU). 
Seções de Imagem Comum (CIG)

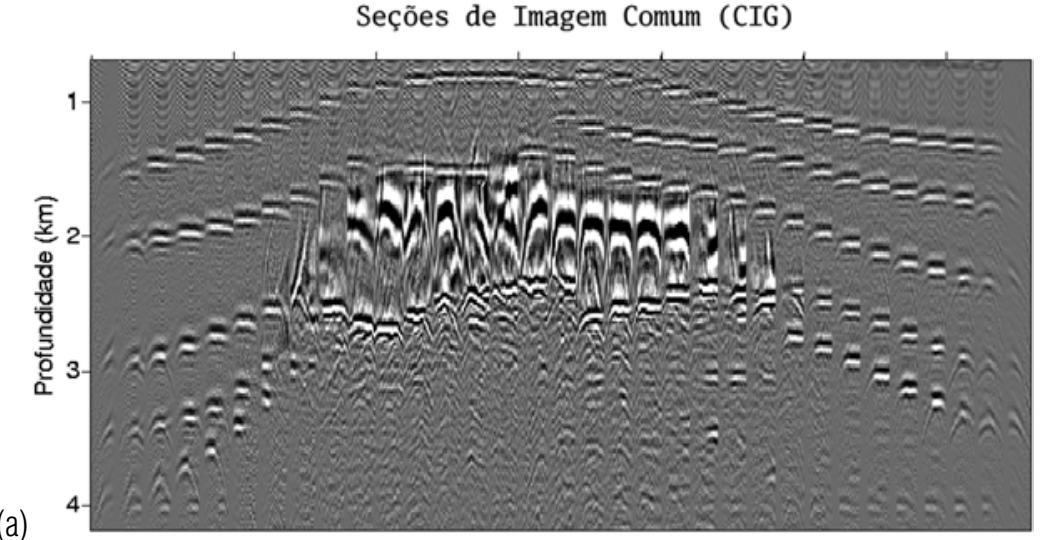

(a)

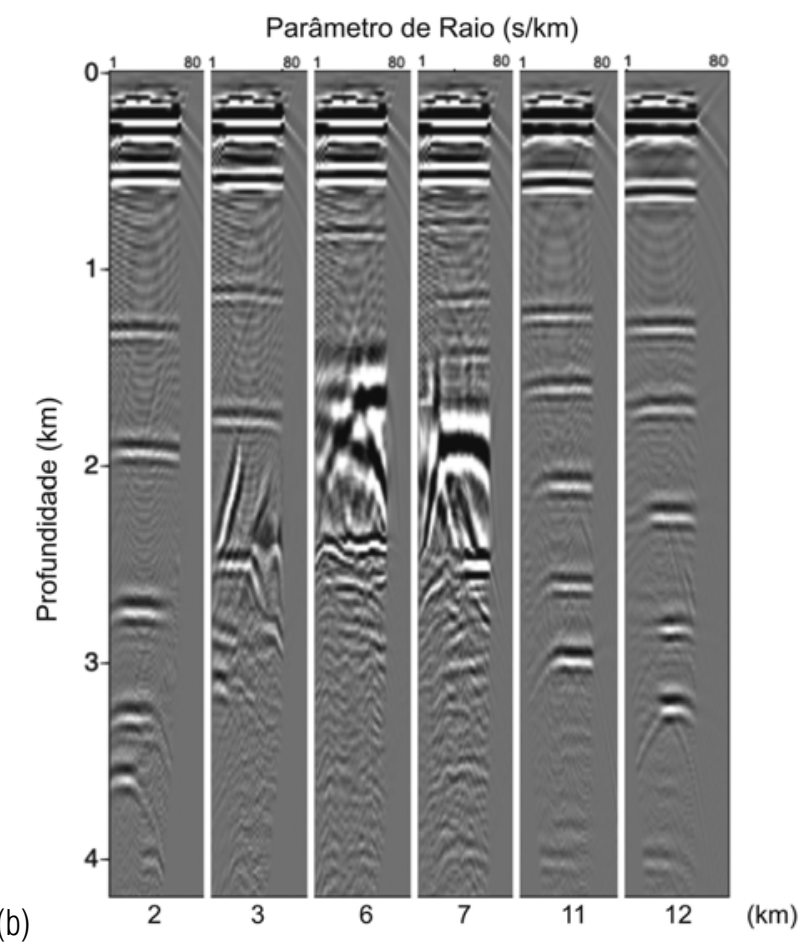

Figura 5 - (a) Seç̃os de imagem comum (CIG), indexadas pelo parâmetro de raio da onda plana na superfície, obtidas a partir do método de migração Kirchhoff de ondas planas. (b) Seções de CIGs nas posições: 2,3,6,7,11 e $12 \mathrm{~km}$ - Cada seção possui 80 parâmetros de raio na faixa de -0,4 a 0,4 s/km.

1501 amostras em cada traço sísmico. 0 menor afastamento é de $100,58 \mathrm{~m}$. A malha do campo de velocidades possui 1000 pontos na direção horizontal (x) por 1185 na vertical (z), com intervalo de amostragem horizontal de 26,67 $\mathrm{m}$ e 6,67 $\mathrm{m}$ na vertical.

A partir dos dados originais geramos, através da transformada "slant-stack" das seções de receptores comuns, 81 seções de parâmetro de raio, na faixa de $-0,64 \mathrm{~s} / \mathrm{km}$ a $0,62 \mathrm{~s} / \mathrm{km}$. Essas seções foram migradas com o nosso método Kirchhoff de ondas planas e o resultado da soma de todas as seções de ondas planas migradas separadamente é apresentado na Figura 6(b). Quantos aos eventos que aparecem na seção migrada, principalmente, os eventos antes do corpo de sal, esses são reais, presentes em outras migrações deste mesmo dado.

De uma forma geral, o resultado obtido com a migração tipo Kirchhoff de ondas planas apresenta uma imagem de boa qualidade, pois mostra uma boa delineação do corpo de sal, conseguindo portanto imagear a base e o topo do corpo de sal e os refletores em torno do mesmo. 


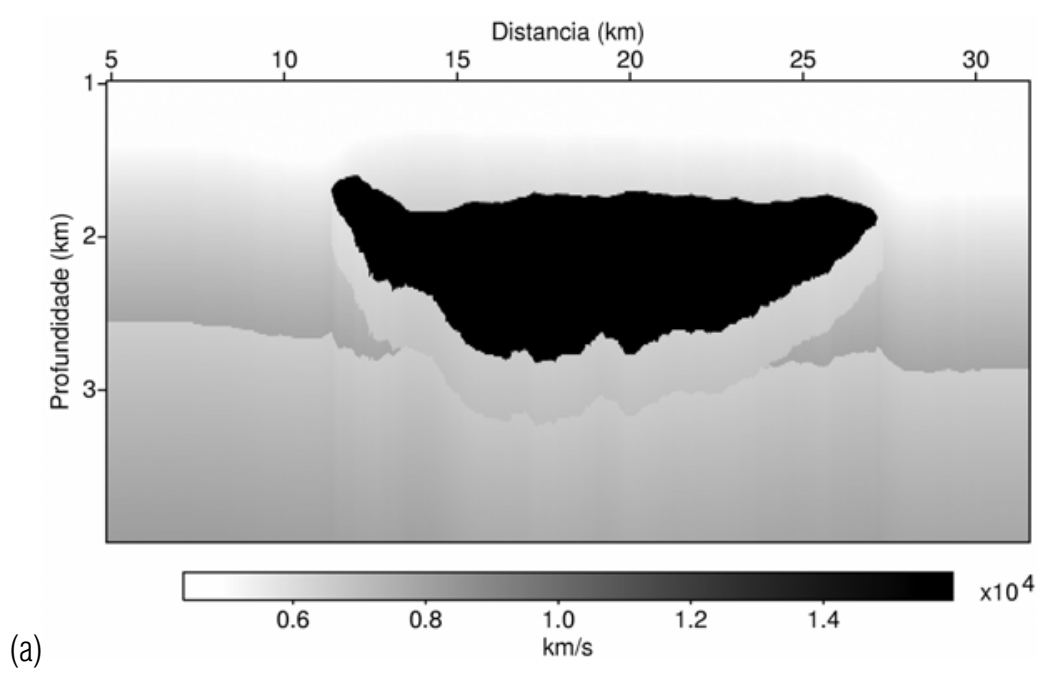

(a)

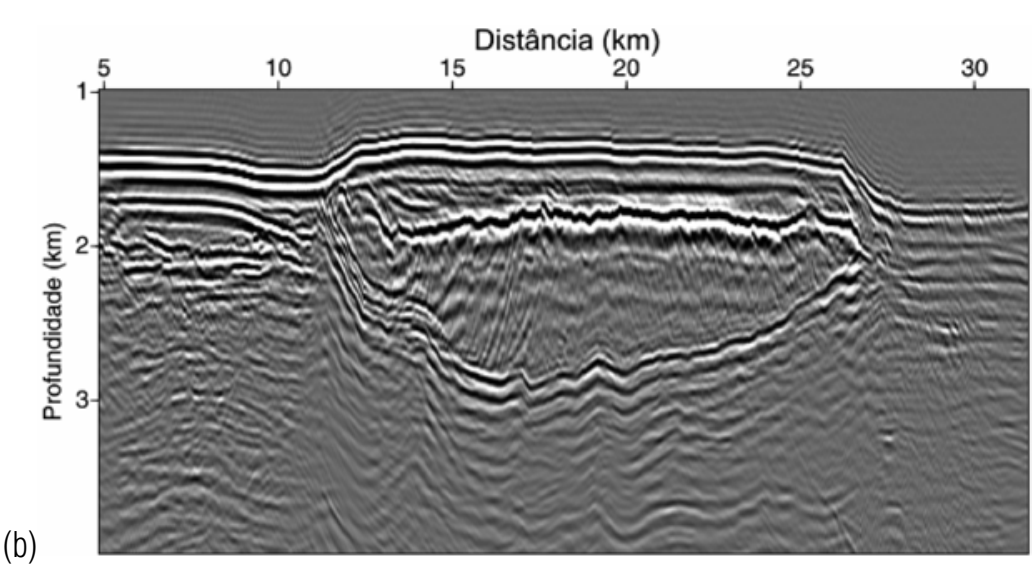

Figura 6 - (a) Modelo de velocidade para os dados do Golfo do México. (b) Resultado da migração do método Kirchhoff de ondas planas após a soma de 81 seções de parâmetro de raio migradas.

\section{CONCLUSÕES}

Neste trabalho apresentamos um método para realizar a migração de dados de fontes de ondas planas em profundidade usando 0 método integral de Kirchhoff.

A análise dos resultados demonstra que 0 método de migração pré-empilhamento em profundidade do tipo Kirchhoff, desenvolvido no domínio das ondas planas, apresenta-se como uma nova alternativa de migração para meios com variação lateral de velocidade. A precisão e a eficiência dessa técnica foram avaliadas comparando-se os resultados obtidos em dados sintéticos com os resultados fornecidos pela migração Kirchhoff convencional de tiro comum.

No modelo SEG-EAGE, que representa uma almofada de sal e suas adjacências, o resultado apresentado pela migração Kirchhoff de ondas planas mostrou uma imagem de boa qualidade e comparável com o resultado da migração Kirchhoff de tiro comum, porém a um menor custo computacional em relação a esta migração. Também os resultados obtidos são bastantes animadores, pois o modelo em profundidade foi quase que completamente reconstituído. Apenas partes dos refletores bem abaixo da almofada de sal não foram recuperadas.

Comparando-se as imagens em profundidade obtidas com as técnicas de migração testadas, para o modelo SEG-EAGE, podemos concluir que em situações de forte contraste de velocidades e mergulhos acentuados, o método de migração Kirchhoff de ondas planas conseguiu posicionar corretamente os refletores, acima do corpo do sal e também em torno do mesmo, gerando uma imagem migrada final de boa qualidade do modelo.

Para os dados reais do Golfo do México, localizados em um ambiente salino, o método também apresentou uma boa imagem migrada mostrando o topo e a base o corpo de sal completamente delineados. 
Portanto, o método Kirchhoff de ondas planas, com seções de ondas planas cuidadosamente selecionadas, consegue produzir imagens comparáveis às fornecidas pelo método Kirchhoff de tiro comum e com uma melhor eficiência computacional. Além disso, podemos ainda destacar que durante 0 processo de migração seções de imagem comum podem ser produzidas, sem nenhum custo computacional extra, e essas seções podem ser utilizadas para avaliar o campo de velocidades usado no processo de migração dos dados.

\section{AGRADECIMENTOS}

Os autores agradecem aos revisores pelos valiosos comentários e sugestões, ao CTPETRO/CNPq-FINEP pelo apoio à rede Cooperativa de Pesquisa em Risco Exploratório, à ANP (Agência Nacional do Petróleo, Gás Natural e Biocombustíveis), PETROBRAS e ao CPGG/UFBA pelo apoio no desenvolvimento deste trabalho. Reynam Pestana agradece ao CNPq pelo suporte, através de bolsa de pesquisa, processo 300.312/88-1 (RM).

\section{REFERÊNCIAS}

AKBAR FE, SEN MK \& STOFFA PL. 1996. Prestack plane-wave Kirchhoff migration in laterally varying media. Geophysics, 61: 1068-1079.

ALDUNATE GC. 2002. Migração pré-empilhamento usando operadores de extrapolação tipo "split-step". Dissertação de Mestrado, Universidade Federal da Bahia. 75 p.

DUQUET B, LAILLY P \& EHINGER A. 2001. 3D Plane wave migration of streamer data. $71^{\text {st }}$ Ann. Mtg., Soc. Expl. Geophysics, 1033-1036.

FARIA EL \& STOFFA PL. 1994. Traveltime computation in transversely isotropic media. Geophysics, 59: 272-281.

HILDEBRAND ST \& CARROLL RJ. 1993. Radon depth migration. Geophysical Prospecting, 41: 229-240.

LIU F, STOLT RRH, HANSON DW \& DAY RS. 2002. Plane wave source composition: an accurate phase encoding scheme for prestack migration. $72^{\text {nd }}$ Ann. Mtg., Soc. Expl. Geophysics, 1156-1159.
MOREIRA NETO CA. 2004. Migração pré-empilhamento em profundidade utilizando ondas planas. Tese de Doutorado, Universidade Federal da Bahia, Salvador, Bahia. 125 p.

MOREIRA NETO CA, PESTANA RC \& ALDUNATE GC. 2005. Migração pré-empilhamento em profundidade no domínio da frequiência de seções de ondas planas. Revista Brasileira de Geofísica, 23(4): 359-370.

MORTON SA \& OBER CC. 1998. Faster shot-record depth migration using phase encoding. $68^{\text {th }}$ Ann. Mtg. Soc. Expl. Geophysics, 11311134.

PESTANA RC, STOFFA PL \& SANTOS JRSB. 2001. Plane wave prestack time migration. JSE, 9: 211-222.

RIETVELD WEA \& BERKOUT AJ. 1994. Prestack depth migration by means of controlled illumination. Geophysics, 59: 801-809.

ROMERO LA, GHIGLIA DC, OBER CC \& MORTOM SA. 2000. Phase encoding of shot records in prestack migration. Geophysics, 65: 426-436.

SCHNEIDER WA. 1978. Integral formulation for migration in two and three dimensions. Geophysics, 43: 49-76.

SCHNEIDER WA Jr., RANZIGER KA, BALCH AH \& KRUSE C. 1992. A dynamic programming approach to first arrival computation in media with arbitrarily distributed velocities. Geophysics, 57: 39-50.

SCHULTZ PS \& CLAERBOUT JF. 1978. Velocity estimation and downward continuation by wavefront synthesis. Geophysics, 43: 691714.

STOFFA PL, BUHL P, DIEBOLD JB \& FRIEDEMANN W. 1981. Direct mapping of seismic data to the domain of intercept time and ray parameter - a plane wave decomposition. Geophysics, 46: 255-267.

ZHANG Y, SUN J, NOTFORS C, GRAY S, CHERNIS L \& YOUNG J. 2003. Delayed-shot 3D prestack depth migration. $65^{\text {th }}$ EAGE Conference \& Exhibition, Extended Abstracts. CD-ROM.

ZHANG Y, SUN J, NOTFORS C, GRAY S, CHERNIS L \& YOUNG J. 2005.

Delayed-shot 3D depth migration. Geophysics, 70: E1-E28.

\section{NOTAS SOBRE OS AUTORES}

Reynam da Cruz Pestana é graduado em Física (UFBA/1983) e Doutor em Geofísica pela UFBA, 1988. Pós-doutorado em Geofísica no Instituto de Geofísica da Universidade de Karlsruhe (Alemanha), 1989-1991, e de 1998-1999 no Instituto de Geofísica da Universidade do Texas em Austin. De 1988 até o presente é pesquisador do CPGG/UFBA, atuando no Grupo de Geofísica de Exploração de Petróleo. Desde 2006 é Professor Associado I do Departamento de Física da Terra e do Meio Ambiente do Instituto de Física e da Pós-graduação em Geofísica da UFBA. Tem atuado no desenvolvimento de métodos e algoritmos de processamento e imageamento sísmicos. Mais recentemente suas pesquisas envolvem técnicas de migração de dados sísmicos decompostos em ondas planas. É membro da SBGf e da SEG.

Celso Alves Moreira Neto é bacharel em Física pela UFMG (1999) e Doutor em Geofísica pela UFBA (2004). Desde 2006 é geofísico da PETROBRAS, Unidade de Negócios de Sergipe - Alagoas (UN-SEAL) onde trabalha com processamento de dados sísmicos. Tem como principal área de interesse o processamento sísmico com ênfase em migração de dados sísmicos. É membro da SBGf. 